\section{Vigilância de eventos adversos \\ a medicamentos em hospitais: \\ aplicação e desempenho de \\ rastreadores}

\section{Surveillance of adverse drug events in hospitals: implementation and performance of triggers}

\author{
Fabíola Giordani ${ }^{\prime \prime \prime}$ \\ Suely Rozenfeld' \\ Daniela Ferreira Miyata de Oliveira" \\ Gelena Lucinéia Gomes da Silva Versa"'I \\ Joelma Suto Terencio"'I \\ Luciane de Fátima Caldeira" \\ Luiz Carlos Gonçalves de Andrade III $^{\prime}$ \\ 'Escola Nacional de Saúde Pública da Fundação Oswaldo Cruz/Ministério da \\ Saúde. \\ " Universidade Estadual do Oeste do Paraná. \\ "I Hospital Universitário do Oeste do Paraná.
}

\section{Resumo}

Os eventos adversos a medicamentos (EAMs) são causa importante de comprometimento da qualidade da atenção ao paciente hospitalizado e, por isso, devem ser identificados e caracterizados. Para tanto surgiram listas de rastreadores, entre elas a proposta pelo Institute for Healthcare Improvement. Aqui é apresentado o processo da aplicação dos rastreadores e o seu desempenho em um hospital de ensino. As informações sobre os rastreadores e os EAM foram coletadas por meio de revisão retrospectiva dos prontuários de pacientes com alta hospitalar de janeiro a junho de 2008. Foram identificados 497 rastreadores em 177 prontuários, onde cada prontuário apresentou, em média, 2,33 ( $\mathrm{DP}=2,7$ ) rastreadores. Os encontrados com mais frequência foram: "antiemético" (72,1/100 prontuários), "interrupção abrupta da medicação” (70,0/100 prontuários) e "sedação excessiva, sonolência, torpor, letargia, queda e hipotensão" (34,6/100 prontuários). Os mais eficientes na captura de EAM (rendimento), isto é, aqueles que uma vez identificados sinalizaram possíveis eventos foram "antagonista de benzodiazepínico", "antidiarréicos" e "rash cutâneo". Os EAM mais encontrados foram relacionados aos rastreadores "interrupção abrupta da medicação” (8,3/100 prontuários), "antiemético" (4,6/100 prontuários) e "rash cutâneo" (2,1/100 prontuários). Essas considerações apontam para a utilidade do emprego da lista de rastreadores e podem contribuir para decidir sobre ajustes na sua aplicação.

Palavras-chave: Monitoramento de Medicamentos. Sistemas de Notificação de Reações Adversas a Medicamentos. Farmacovigilância. Preparações Farmacêuticas. Efeitos adversos. Hospitais. Controle de Formulários e Registros. Hospitalização.

Apoio Financeiro: Este estudo contou com o apoio financeiro do Conselho Nacional de Pesquisa através do Processo n 471685/2007-0 e da bolsa de doutorado concedida à primeira autora. Correspondência: Fabíola Giordani. Universidade Estadual do Oeste do Paraná , Centro de Ciências Médicas e Farmacêuticas - Colegiado de Farmácia. Rua Universitária, 2069 Bairro Jardim Universitário - CEP 85819-110 Cascavel, PR. E-mail: fabiola.giordani@yahoo.com.br 


\section{Abstract}

Adverse drug events (ADE) are important causes of impairment of the quality of care for inpatients and therefore should be identified and characterized. Thus, lists of triggers were developed, including the proposal by the Institute for Healthcare Improvement. We analyzed the application of these triggers in a university hospital, with the cooperation of pharmacy and medical students, field training, standardized forms and manuals. Here, we present the process of application and performance of these triggers in a teaching hospital. The information on the triggers and $\mathrm{ADE}$ were collected through a retrospective chart review of patients discharged from January to June 2008. Four hundred and ninety-seven triggers were identified in 177 charts, and each chart had $2.33(\mathrm{SD}=2.7)$ triggers on average. The most frequent triggers were: "antiemetic" (72.1/100 charts), "abrupt cessation of medication" (70.0/100 charts) and "over-sedation, drowsiness, numbness, lethargy, hypotension and fall” (34.6/100 charts). The most effective triggers for capturing ADE were "benzodiazepine antagonist", "antidiarrheal" and "rash", which, when identified in charts, were related to an event. The ADE most commonly found were related to the triggers, "abrupt cessation of medication" (8.3/100 charts), "antiemetic" (4.6/100 charts), "rash" and "anti-allergy" (2.1/100 charts). These results may help to decide which triggers are more useful in each situation.

Keywords: Drug Monitoring. Adverse Drug Reaction Reporting Systems. Pharmacovigilance. Pharmaceutical Preparations. Adverse effects. Hospitals. Forms and Records Control. Hospitalization.

\section{Introdução}

Os eventos adversos a medicamentos (EAMs) estão entre as causas mais frequentes de problemas ocorridos durante o processo de cuidado no ambiente hospitalar ${ }^{1,2}$. Estima-se que $14,8 \%$ a $59 \%$ deles poderiam ser evitados ${ }^{3}$. Assim, sua identificação durante a internação hospitalar é fundamental para auxiliar o desenvolvimento de estratégias de aprimoramento da qualidade da atenção à saúde.

Um dos métodos mais usados para identificar EAM no ambiente hospitalar é a notificação voluntária, cuja principal limitação é a subnotificação dos casos. Estima-se que o método detecte apenas um em cada vinte eventos ocorridos ${ }^{4}$. Por isso, surgiram outras abordagens visando conferir maior eficiência na identificação dos EAM e aumento da segurança do paciente. Além disso, a associação de técnicas de identificação de EAM é prática comum e recomendada por vários autores ${ }^{5,6}$.

A revisão retrospectiva de prontuários é outra abordagem para identificar EAM; entretanto, tem o inconveniente de demandar, em excesso, tempo e recursos humanos ${ }^{7}$. Como estratégia a ser massificada, pode não ser viável para monitorar a prática assistencial ${ }^{8}$.

O Institute for Healthcare Improvement (IHI) desenvolveu um método alternativo à revisão tradicional de prontuários, que emprega 19 rastreadores, incluindo medicamentos, parâmetros laboratoriais e informações sobre o cuidado e a evolução clínica do paciente ${ }^{9}$. Os rastreadores de EAM foram empregados em investigações no Brasil ${ }^{10,11}$, na Europa ${ }^{8}$ e incorporados em ações de monitoramento nos Estados Unidos ${ }^{12,13}$. O objetivo do artigo é oferecer elementos para o emprego de rastreadores na vigilância dos eventos adversos a medicamentos em hospitais, apresentando o processo de aplicação de uma lista de rastreadores, assim como o desempenho de cada um deles, em um hospital público geral. 


\section{Método}

Aplicou-se técnica de revisão retrospectiva a uma amostra de prontuários, usando uma lista de rastreadores previamente testada ${ }^{9}$ (Quadro 1). Estudantes de medicina e de farmácia, e profissionais de saúde, foram treinados para a extração de informações, usando instrumentos padronizados e manual contendo instruções de preenchimento, definições relevantes e orientações para avaliar a ocorrência de EAM. A seguir, procederam-se os ajustes na lista de rastreadores, considerando a viabilidade e a adequação dos mesmos à realidade local. Os ajustes foram efetuados por equipe multiprofissional, composta por um médico clínico, duas enfermeiras e três farmacêuticas, supervisionados por uma médica com formação em saúde pública.

A revisão dos prontuários desenvolveu-se nas seguintes etapas:

- Identificação dos rastreadores: os prontuários foram revisados por estudantes de graduação de farmácia e de medicina, do quarto ou do quinto ano, para a bus- ca de rastreadores, iniciando-se pelos parâmetros laboratoriais presentes nos laudos. Os medicamentos foram identificados nas folhas de prescrição, e os demais rastreadores nos registros das equipes médica e de enfermagem. Essa ordem é a recomendada pelo $\mathrm{IHI}^{9}$.

- Identificação dos EAMs: os prontuários com rastreadores foram reavaliados, de modo independente, por um enfermeiro e um farmacêutico, para verificar a presença de um possível EAM.

- Reunião de consenso: os prontuários dos pacientes com possíveis EAM foram submetidos à discussão, em reunião com os membros da equipe multiprofissional de avaliação. Nessa etapa foram discutidas as divergências em relação à ocorrência de EAMs, e suas classificações quanto à causalidade e ao grau do dano, até chegar ao consenso.

\section{Definição de EAM}

Qualquer dano, grave ou leve, causado pelo uso de um medicamento com

Quadro 1 - Rastreadores propostos pelo Institute of Healthcare Improvement (IHI), $2004^{9}$.

Chart 1 - Triggers proposed by the Institute of Healthcare Improvement (IHI), $2004^{9}$.

\begin{tabular}{|l|}
\hline \multicolumn{1}{|c|}{ Relação dos rastreadores } \\
\hline Difenitramina \\
Vitamina K \\
Flumazenil \\
Antiemético (droperidol, ondansetrona, prometazina, hidroxizina, trimetobenzamida, \\
proclorperazina ou metoclopramida) \\
Naloxona \\
Antidiarréicos \\
Poliestireno de sódio \\
Glicose $<50$ \\
Exame positivo para C. difficile nas fezes \\
Tempo de tromboplastina parcialmente ativada (PTT) $>100$ segundos \\
Razão Normalizada Internacional (IRN) $>6$ \\
Contagem de glóbulos brancos $<3.000$ \\
Contagem de plaquetas $<50.000$ \\
Níveis de digoxina $>2 n g / m L$ \\
Elevação da creatinina sérica \\
Sedação excessiva, letargia, queda \\
Rash \\
Interrupção abrupta da medicação \\
Transferência para nível de cuidado mais complexo
\end{tabular}


finalidade terapêutica. Essa definição engloba reações adversas a medicamentos e erros de medicação ${ }^{14}$.

\section{Local do estudo e população}

O estudo foi desenvolvido em um hospital de ensino localizado na região Oeste do Estado do Paraná. O hospital possui 173 leitos exclusivos do SUS para atendimentos de nível secundário e terciário, com diversas especialidades clínicas e cirúrgicas. O projeto foi aprovado pelo Comitê de Ética em Pesquisa institucional (Parecer 039/2009CEP) e não apresenta qualquer conflito de interesse real ou potencial.

A população incluída constituiu-se de pacientes com 15 anos ou mais de idade, com alta hospitalar em seis meses consecutivos. Foram excluídas as internações obstétricas, aquelas com duração de até 48 horas ou de períodos em unidade de terapia intensiva ou de pronto atendimento. O método foi testado em uma amostra piloto de 10 prontuários, e posteriormente aplicado a uma amostra aleatória simples de 240 prontuários de pacientes com alta hospitalar entre janeiro e junho de 2008, pertencente à base de dados de um estudo sobre EAM. A amostra foi extraída dos bancos de dados das internações dos meses selecionados e calculada considerando nível de significância estatística de 95\%, proporção de EAM de 15\% e precisão absoluta de $10 \%$.

\section{Extração de informações e variáveis}

A extração de informações dos prontuários foi realizada com coleta e avaliação duplicadas nas diversas etapas, e com registro de todos os medicamentos prescritos/usados, em Ficha de Coleta de Medicamentos (Figura 1). Nela, cada medicamento foi adicionado a uma linha, e cada dia de internação a uma coluna. As células foram preenchidas de acordo com a condição do medicamento, ou seja, se uma vez prescrito ele foi usado ou não.

As variáveis extraídas do prontuário foram referentes às características demográficas, sociais e de saúde do paciente, assim como da internação. No primeiro grupo estão: sexo (feminino, masculino), raça/cor da pele (branco, negro, pardo, outro), ocupação, idade (em anos de vida), diagnósticos principal e secundários e co-morbidades registradas. E no segundo grupo: caráter da internação (urgência/emergência, eletiva), resultado do cuidado (alta hospitalar, transferência, óbito), internação em UTI (sim, não), tipo de atendimento

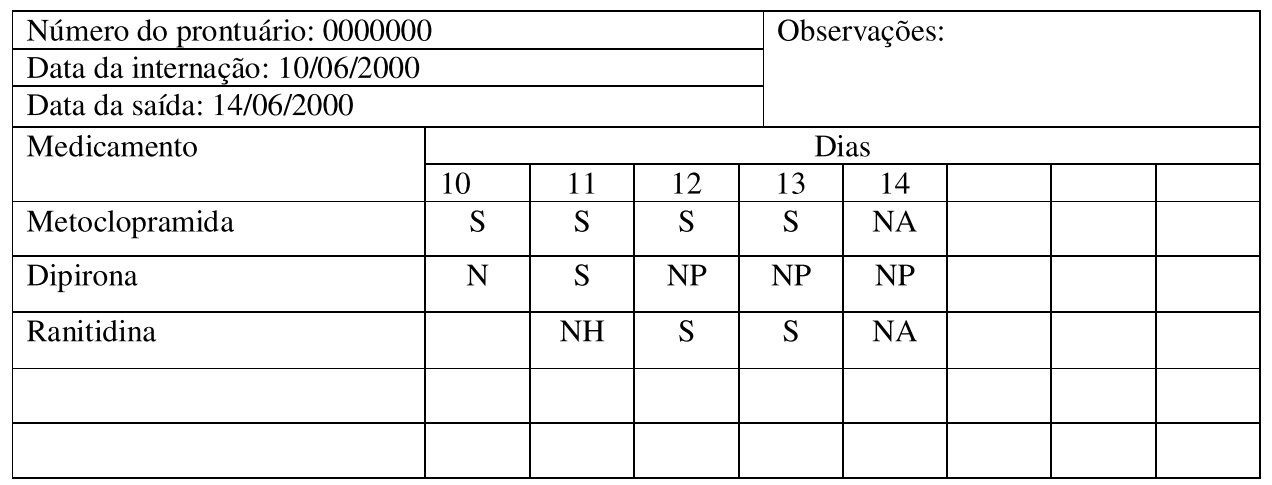

$\mathrm{S}$ - medicamento prescrito e administrado; $\mathrm{N}$ - medicamento prescrito se necessário e não administrado; $\mathrm{NH}$ - Medicamento prescrito, mas não há na farmácia; NA - Medicamento prescrito e não administrado (razão desconhecida); NP - Medicamento prescrito que deixou de sê-lo.

$S$ - medication prescribed and administered; $N$ - medication prescribed PRN and not administered; $N H$ - medication prescribed, but unavailable at the pharmacy;NA - medication prescribed and not administered (unknown reason); NP- medication prescribed and then discontinued.

Figura 1 - Esquema representativo da Ficha de Coleta de Medicamentos (FCM).

Figure 1 - Representation of Drug Collection Form (FCD). 
(cirúrgico, clínico, ambos), duração e custo da internação.

\section{Análises}

A probabilidade de associação entre o uso de medicamentos e o evento adverso foi definida a partir das informações da literatura especializada e das seguintes questões: $\mathrm{O}$ evento pode estar associado ao medicamento suspeito, considerando-se as suas propriedades? O evento pode estar associado ao medicamento suspeito, considerando-se a condição clínica do paciente? $\mathrm{O}$ evento pode estar associado ao medicamento suspeito, considerando-se a relação temporal entre sua utilização e o evento?
Os eventos adversos foram classificados quanto à causalidade em definido, provável, possível e duvidoso, segundo o Algoritmo de Naranjo ${ }^{15}$, e quanto ao grau de dano, segundo o critério do $\mathrm{IHI}^{9}$ (Figura 2).

O desempenho dos rastreadores e a sua capacidade de captar eventos adversos aos medicamentos foram analisados em três componentes. O primeiro deles foi calculado dividindo-se o número de registros de cada rastreador pelo total de prontuários avaliados, multiplicado por 100 (1); o segundo, dividindo o número de EAM identificados pelos rastreadores pelo total de prontuários avaliados, multiplicado por 100 (2); e o terceiro foi calculado dividindo-se (2) por (1) e multiplicando por 100. Este último é uma proporção que define o rendimento

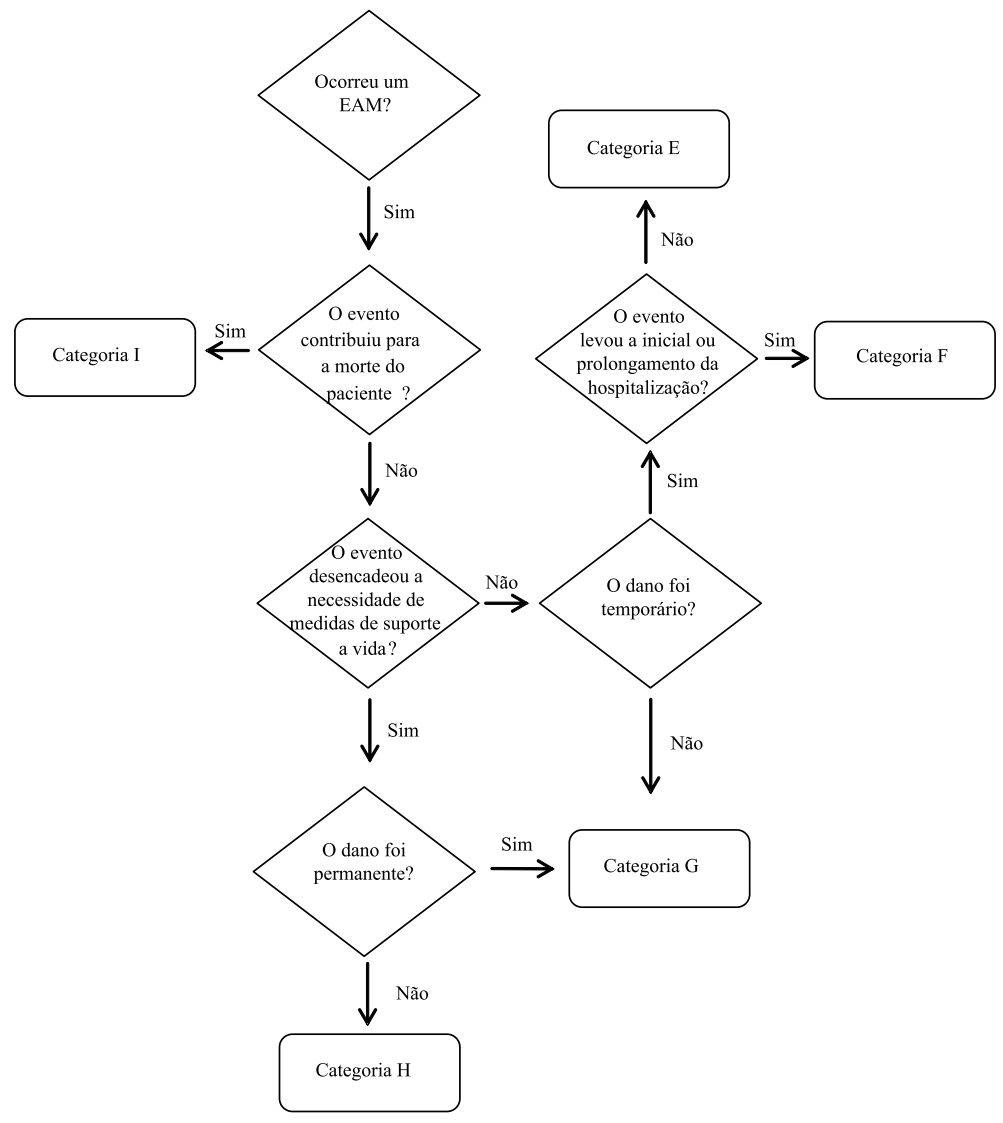

NCCMERP Index for Classifying Medication Errors modified Algorithm

Figura 2 - Fluxograma modificado para estabelecimento do grau de dano dos Eventos Adversos a Medicamentos (EAMs).

Figure 2 - Flowchart modified to establish the category of harm from adverse drug event (ADE). 
do rastreador e expressa, em valores relativos, o potencial de cada um deles para identificar EAM.

Os dados coletados foram digitados em um banco desenvolvido no programa Epidata $3.0^{16}$, em duplicata, de forma independente. As divergências, identificadas pelo relatório de validação, foram revistas e resolvidas pela primeira autora do artigo.

\section{Resultados}

Foram identificados 497 rastreadores em 177 prontuários. A média foi 2,33 (DP = $2,7)$ rastreadores por prontuário, sendo que $62,9 \%$ deles possuíam entre 1 e 4 (Tabela 1). Os rastreadores propostos pelo $\mathrm{IHI}^{9}$ e ajustados à realidade local estão apresentados na Tabela 2. Após os ajustes foram empregados 19 rastreadores para a detecção de EAM, dos quais oito relacionados à medicação, sete aos exames laboratoriais, três à evolução dos pacientes e um, simultaneamente, relacionado à medicação e à evolução clínica do paciente.

A Tabela 2 mostra o desempenho de cada um dos rastreadores. Os mais encontrados na amostra foram: "antieméticos" (72,1/100 prontuários), "interrupção abrupta da medicação” (70,0/100 prontuários) e "sedação excessiva, sonolência, torpor, letargia, queda, hipotensão" (34,6/100 prontuários). Doze dos 19 rastreadores levaram a possíveis EAM, sendo os rastreadores que identificaram número mais elevado de eventos "interrupção abrupta da medicação" (8,3/100 prontuários), "antiemético" (4,6/100 prontuários), "rash cutâneo"
(2,1/100 prontuários), "antialérgicos" (1,7/100 prontuários) e "sedação excessiva, sonolência, torpor, letargia, queda, hipotensão" (1,7/100 prontuários). Observou-se que 4/20 EAM sinalizados por "interrupção abrupta da medicação" foram considerados com grau de dano mais elevado (F e H. Ver Figura 2). Por outro lado, 11/11 EAM sinalizados pelo uso de "antieméticos" foram classificados com grau de dano menor (E. Ver Figura 2).

Quanto ao rendimento dos rastreadores, podemos identificar três grupos de rastreadores. O primeiro é constituído por aqueles com melhor rendimento: "antagonista de benzodiazepínico", "antidiarréicos" e "rash cutâneo", que, em 100\% dos casos, sinalizaram um possível EAM (Tabela 2). No caso dos dois primeiros, os eventos foram considerados com grau de dano mais elevado ( $\mathrm{H} \mathrm{e} \mathrm{F}$, respectivamente. Ver Figura 2) e, no terceiro, com grau de dano menor (E. Ver Figura 2). Os eventos considerados graves foram intoxicação por midazolam, com necessidade de medicação de resgate; e diarréia intensa, com administração de loperamida e interrupção do antimicrobiano sultamicilina. Já os eventos de menor grau de dano, relacionados com o rastreador "rash cutâneo", foram cinco casos de erupções cutâneas do tipo alérgico e quatro reações locais pós-administração de ranitidina.

No segundo grupo encontram-se rastreadores com rendimento intermediário: "KPTT > 100 segundos", "antialérgicos", "plaquetas $<50.000$ " e "glicemia $<50 \mathrm{mg} / \mathrm{dL}$ ", com sensibilidade entre $30 \%$ e $70 \%$ para

Tabela 1 - Distribuição dos rastreadores, segundo número de rastreadores encontrados, em hospital de ensino do oeste do Paraná, 2008.

Table 1 - Distribution of triggers, according to number of triggers found in a teaching hospital in Western Paraná, 2008.

\begin{tabular}{ccc}
\hline Número de rastreador & Número de prontuários & Proporção (\%) \\
\hline 0 & 63 & 26,2 \\
$1-4$ & 151 & 62,9 \\
$5-10$ & 21 & 8,8 \\
$11-25$ & 5 & 2,1 \\
\hline
\end{tabular}


Tabela 2 - Frequência de rastreadores (R) e de eventos adversos a medicamentos (EAMs) por 100 prontuários* e rendimento de cada rastreador, em hospital de ensino do oeste do Paraná, 2008.

Table 2 - Frequency of triggers (T) and adverse drug events (ADE) per 100 charts* and performance of each trigger in a teaching hospital in Western Paraná, 2008.

\begin{tabular}{|c|c|c|c|}
\hline Lista de rastreadores & $\begin{array}{c}\text { R por } 100 \\
\text { prontuários (1) }\end{array}$ & $\begin{array}{l}\text { EAM por } 100 \\
\text { prontuários (2) }\end{array}$ & $\begin{array}{c}\text { Rendimento relativo } \\
\text { do rastreador } \\
(3)=(2) /(1) \times 100\end{array}$ \\
\hline $\begin{array}{l}\text { Antialérgicos (dexclorfeniramina, loratadina, prometazina } \\
\text { e epinefrina) }\end{array}$ & 2,9 & 1,7 & 57,1 \\
\hline Coagulantes (vitamina K e protamina) & 3,3 & 0,4 & 12,5 \\
\hline Antagonista de benzodiazepínico (flumazenil) & 0,4 & 0,4 & 100,0 \\
\hline $\begin{array}{l}\text { Antiemético (bromoprida, metoclopramida e } \\
\text { ondansetrona) }\end{array}$ & 72,1 & 4,6 & 6,4 \\
\hline Antagonista de opióide (naloxona) & 0,0 & 0,0 & 0,0 \\
\hline Antidiarréicos (loperamida) & 0,4 & 0,4 & 100,0 \\
\hline Resina de troca iônica (poliestirenossulfonato de cálcio) & 0,0 & 0,0 & - \\
\hline Glicemia $<50 \mathrm{mg} / \mathrm{dL}$ & 1,3 & 0,4 & 33,3 \\
\hline Exame positivo para $C$. difficile nas fezes & 0,0 & 0,0 & - \\
\hline KPTT $>100$ segundos & 1,3 & 0,8 & 66,7 \\
\hline $\mathrm{RNI}>6$ & 0,4 & 0,0 & 0,0 \\
\hline Leucócitos $<3.000$ & 2,1 & 0,0 & 0,0 \\
\hline Plaquetas $<50.000$ & 2,1 & 0,8 & 40,0 \\
\hline $\begin{array}{l}\text { Uso de digoxina e arritmia, bradicardia, náuseas, vômito, } \\
\text { anorexia ou alterações visuais }\end{array}$ & 0,0 & 0,0 & - \\
\hline Elevação da creatinina sérica & 6,3 & 0,4 & 6,7 \\
\hline $\begin{array}{l}\text { Sedação excessiva, sonolência, torpor, letargia, queda, } \\
\text { hipotensão }\end{array}$ & 34,6 & 1,7 & 4,9 \\
\hline Rash cutâneo & 2,1 & 2,1 & 100,0 \\
\hline Interrupção abrupta da medicação & 70,0 & 8,3 & 11,9 \\
\hline Transferência para nível de cuidado mais complexo & 7,9 & 0,0 & 0,0 \\
\hline
\end{tabular}

${ }^{*}$ número de prontuários $=240 /{ }^{*}$ number of charts $=240$

captar eventos, embora com frequência absoluta abaixo de 2,1 rastreadores por 100 prontuários. O KPTT e os antialérgicos identificaram eventos com grau de dano menor (E. Ver Figura 2), a contagem de plaquetas e os níveis séricos de glicose com grau de dano mais elevado ( $\mathrm{F} \mathrm{e} \mathrm{H}$, respectivamente. Ver Figura 2). Em um dos casos, a plaquetopenia foi identificada com presença de melena. Já o evento de maior gravidade foi um caso de hipoglicemia grave e prolongada, com duração aproximada de seis horas.

No terceiro grupo de rastreadores estão os que apresentam rendimento abaixo de
$30 \%$. São eles: "coagulantes" (12,5\%), "interrupção abrupta da medicação" (11,9\%), "elevação da creatinina sérica" $(6,7 \%)$, "antiemético" (6,4\%) e "sedação excessiva, sonolência, torpor, letargia, queda, hipotensão" (4,9\%). Destacam-se algumas características, entre elas o fato de o uso de coagulantes ter permitido identificar um EAM com grau de dano elevado, onde o paciente apresentou hemorragia (H. Ver Figura 2). Além disso, a interrupção da medicação e o uso de antiemético permitiram captar mais eventos, 13 e 11 EAMs, respectivamente, já que são os rastreadores encontrados com maior frequência. 


\section{Discussão}

\section{Aplicação dos rastreadores}

A seguir estão apresentadas algumas considerações relevantes sobre o processo de ajuste e aplicação dos rastreadores.

Entre os rastreadores relacionados aos medicamentos, alguns deles sofreram acréscimos para esclarecimentos, mais do que ajustes. Aos antieméticos e antidiarréicos foram adicionados os nomes dos fármacos da lista padronizada no Hospital (bromoprida, metoclopramida e ondansetrona, e loperamida, respectivamente). Para os rastreadores "flumazenil" e "naloxona”, foram adotadas, respectivamente, as terminologias antagonistas dos benzodiazepínicos e antagonistas dos opióides. Quanto à "difenidramina”, que pode indicar a ocorrência de reação alérgica relacionada ao uso de medicamentos, foi substituída por "antialérgicos" e acrescentados os nomes dos fármacos padronizados (dexclorfeniramina, loratadina, prometazina, epidefrina). O mesmo ocorreu com o rastreador "vitamina K", que pode sinalizar danos relacionados a sangramento devido ao uso de medicamentos, sendo ele substituído por "coagulantes", uma vez que, no hospital, a protamina também é padronizada. O rastreador "poliestireno de sódio" pode identificar casos nos quais a hipercalemia grave pode ser relacionada ao uso de fármacos, tais como inibidores da enzima conversora de angiotensina, glicosídeos cardíacos, diuréticos poupadores de potássio ou antiinflamatórios não esteroidais; ele foi substituído por "resina de troca iônica" e acrescentado poliestirenossulfonato de cálcio.

O rastreador "interrupção abrupta da medicação" foi considerado presente mediante o registro, em prontuário, da palavra "suspensão", ou quando o medicamento foi suspenso sem causa descrita. Não foram considerados casos de suspensão abrupta: mudança de dose, da via de administração ou do número de doses/dias; medicamento prescrito "se necessário" ou a critério médico e não administrado; final de tratamento, por exemplo, com terapia ou profilaxia antimicrobiana; razões administrativas, como falta do medicamento na farmácia ou ausência de registro por alguns dias. Com relação à mudança de dose ou da via de administração, é preciso ressaltar que a exclusão pode diminuir a especificidade do rastreador, pois pode haver casos em que essas condições estão associadas à EAM. Entretanto, esse evento pode ser captado por outro rastreador. A "interrupção abrupta da medicação" foi o rastreador mais problemático, com o maior número de divergências entre os revisores, pois sua avaliação implica julgamento. É preciso identificar o motivo pelo qual a medicação foi descontinuada e/ou substituída, informação nem sempre disponível no prontuário.

Quanto aos resultados de exames laboratoriais, a alteração mais relevante foi no rastreador "níveis de digoxina", uma vez que, no hospital, tal exame não é realizado rotineiramente. Sendo assim, ele foi alterado para "uso de digoxina" associado às manifestações clínicas de intoxicação por esse fármaco.

A "elevação da creatinina sérica" foi mantida e estabeleceu-se que seria considerada rastreador positivo sempre que houvesse duas aferições consecutivas de creatinina, com aumento de $0,5 \mathrm{mg} / \mathrm{dL}$ entre elas, independente de os valores estarem dentro dos níveis considerados normais ${ }^{17}$.

Os parâmetros dos exames laboratoriais para os rastreadores glicemia $(<50 \mathrm{mg} / \mathrm{dL})$, tempo de tromboplastina parcial ativada (KPTT > 100 segundos), razão normalizada internacional (RNI $>6$ ), leucócitos $(<3.000)$ e plaquetas $(<50.000)$ foram mantidos. Entende-se que valores como os propostos talvez sejam pouco sensíveis para rastrear EAM, pois valores menos extremos podem estar associados à ocorrência dos eventos adversos. Entretanto, eles foram mantidos porque sinalizam a ocorrência de danos graves que poderiam ter sido interceptados antes de atingidos tais valores.

Ao rastreador "sedação excessiva, letargia, queda e hipotensão" foram adicionados os termos sonolência e torpor, mais usados 
no local do estudo. Nesse momento houve dois desafios. O primeiro, referente ao uso indiscriminado da palavra sonolência nos prontuários. Padronizou-se que sonolência só seria considerada rastreador se estivesse registrada no prontuário em pelo menos dois momentos distintos em um período de 24 horas. O segundo desafio foi quanto à "hipotensão". Padronizou-se que "hipotensão" seria rastreador sempre que houvesse registro no prontuário da palavra "hipotensão” ou de valores de pressão arterial iguais ou inferiores a 90/60 mmHg, acrescidos de algum sinal ou sintoma.

Em relação ao rastreador "rash cutâneo", manteve-se o termo em inglês, amplamente usado entre nós, embora nem sempre ele seja registrado como tal nos prontuários. Assim, padronizou-se que o registro de erupção cutânea seria considerado rastreador positivo (presença de "rash cutâneo"), o que reduziria os falsos negativos. Por outro lado, a leitura sistemática dos prontuários, implícita no processo de rastreamento, permitiria identificar patologias cutâneas diagnosticadas e evitaria confundi-las com rastreadores ou com eventos adversos, reduzindo desta forma os falsos positivos. Acredita-se, com isso, ter melhorado a validade da informação.

\section{Desempenho dos rastreadores}

Quanto ao desempenho de cada rastreador, inicialmente o número de rastreadores, por tipo, a cada 100 prontuários, deve ser examinado, já que expressa a carga de trabalho a ser incorporada no processo de identificação de EAM, pois quanto maior o número de prontuários com rastreadores, maior o número daqueles que serão avaliados quanto à presença de eventos. Outra questão está relacionada às dificuldades envolvidas na identificação de cada rastreador e que dependem da natureza de cada um deles. Observa-se que identificar o uso de antieméticos é tarefa simples, ainda que as prescrições sejam manuais e não eletrônicas. Entretanto, além dos antieméticos, os outros dois identificados com mais frequência (interrupção abrupta e sedação) envolvem complexidade, julgamento e definições adicionais.

Ainda na Tabela 2 observa-se o número de EAM - captados pelos rastreadores, um a um - por 100 prontuários. Esse indicador expressa, em termos absolutos, a capacidade de cada rastreador identificar, ou não, EAM. Nesse sentido, 12/19 rastreadores levaram a possíveis EAM, o que traduz, em certa medida, a utilidade do método em seu conjunto; é possível que os outros sete (antagonista de opióide, resina de troca iônica, exame positivo para C. difficile nas fezes, RNI $>6$, leucócitos $<3.000$ e uso de digoxina + sintomas, transferência para nível de cuidado mais complexo) fossem pistas úteis em amostras maiores. O tamanho amostral $(\mathrm{n}=240)$ foi calculado levando-se em conta, apenas, a incidência global de eventos. Entretanto, outros elementos devem ser considerados. A ausência de uso do antagonista de opióide pode ser causada por dificuldade para diagnosticar intoxicações por opióides ou, contrariamente, tanto o diagnóstico como a intervenção serem tempestivos, prescindindo do uso de antagonistas. Quanto à ausência de intoxicação digitálica, supõe-se que o controle do uso de digoxina em pacientes internados esteja sendo efetuada de modo adequado.

Os rastreadores que identificaram número mais elevado de eventos eram esperados, já que estão relacionados aos rastreadores mais encontrados. Outro ponto a considerar é o fato de o grau de dano associado aos eventos captados pelos rastreadores ser distinto; por exemplo, náuseas e vômitos, identificados pelo rastreador uso de antieméticos, normalmente podem ser considerados eventos de baixa gravidade, ao passo que colite pseudomembranosa, identificada pela suspensão de uso de antimicrobianos, pode ser considerado evento de gravidade moderada. A interrupção abrupta da medicação foi associada a um grau de dano mais elevado (F e H. Ver Figura 2). Apesar da dificuldade para avaliar se a interrupção de uma medicação está ou não relacionada a ajustes de 
posologia, suspensão prevista ou de ordem administrativa, esse rastreador é útil, já que permite identificar maior número de eventos e de gravidade diversificada.

Na Tabela 2 está apresentado o rendimento dos rastreadores, medida que expressa o potencial relativo de cada rastreador, comparado aos demais, para identificar eventos.

Há autores ${ }^{18}$ que, ao calcular a proporção de EAM identificados por cada um dos rastreadores, denominam o resultado de valor preditivo positivo (VPP). Entretanto, o conceito pareceria mais apropriado caso os resultados alcançados na identificação de EAM por rastreador fossem confrontados com aqueles alcançados com outros métodos, por exemplo, testes laboratoriais ou auditorias de prontuários.

\section{Virtudes e limitações}

Como virtudes do processo empregado destacam-se: seleção dos prontuários por amostra aleatória simples; extração de informações duplicada; incorporação de acadêmicos na coleta de dados; coleta padronizada dos medicamentos prescritos/usados, dia a dia, durante o período de internação.

De acordo com o $I H I^{9}$ (2004) uma amostra aleatória mensal de 20 prontuários é suficiente para o monitoramento. Neste estudo optou-se pelo uso de amostra aleatória simples mensal que, por ser um plano de amostragem estatisticamente representativo, permite fazer inferências sobre a população de pacientes hospitalizados.

Todas as etapas de extração e análise de rastreadores e de EAM foram efetuadas em duplicata. Tal procedimento não teve como objetivo análise de confiabilidade, mas sim a complementaridade das informações extraídas. Esse procedimento foi importante para contornar a subjetividade nas várias etapas. Deu suporte a essa opção o fato de que, para identificação de eventos adversos por revisão retrospectiva de prontuários, a concordância inter-avaliadores entre pares de médicos é considerada baixa, com um Kappa de 0,25 (IC: 0,05 - 0,45) ${ }^{19}$.
No presente estudo, a identificação dos rastreadores foi realizada por acadêmicos dos cursos de farmácia e de medicina, o que possibilitou otimização do tempo dos profissionais e contribuiu para a formação dos alunos.

O emprego da Ficha de Coleta de Medicamentos (Figura 1) permitiu visualizar, de forma sintética, todos os medicamentos usados pelo paciente durante a internação, dispostos de modo a favorecer a análise da temporalidade, tanto para a avaliação do EAM quanto para a determinação de rastreadores, em especial a interrupção abrupta de medicamentos. Assim, supõe-se ter aumentado a validade das informações sobre as exposições aos fármacos.

A identificação inicial dos rastreadores é uma etapa mais objetiva do método que, inclusive, pode ser realizada de forma automatizada ${ }^{20}$. O monitoramento computadorizado de EAMs é uma técnica promissora. $\mathrm{O}$ uso de programas computadorizados, com rastreadores para identificação de eventos, foi o ponto de partida para o desenvolvimento da ferramenta de monitoramento do $I H I^{2}$. Entretanto, esse método pode implicar em altos custos e ter prontuário digitalizado como requisito $^{13}$. Alguns hospitais brasileiros possuem o prontuário eletrônico, útil para o exame preliminar dos rastreadores. A informatização da prescrição e dos exames laboratoriais pode ser um passo importante para tornar a técnica mais custo-efetiva e as informações mais válidas. Além disso, o tempo economizado seria empregado de modo mais útil para a investigação de rastreadores de maior complexidade. Por exemplo, a interrupção abrupta de medicamentos, cuja análise pode ser inviável para ser efetuada de forma eletrônica.

A escassa disponibilidade de tempo dos profissionais envolvidos na avaliação dos prontuários, especialmente para encontrar um horário comum para a reunião de consenso, sugere a necessidade de se buscar alternativas como a coleta automática de parte dos rastreadores.

Um dos problemas identificados, comum a todos os estudos de natureza 
retrospectiva, foi a baixa qualidade dos registros nos prontuários dos pacientes, especialmente em relação à legibilidade, ao uso de abreviaturas e à insuficiência das informações nas folhas de evolução médica. Essa é uma limitação que ocorre especialmente em relação à documentação dos EAMs, o que pode dificultar a sua caracterização ${ }^{7}$. Como resultado, são apresentadas estimativas menores e menos válidas. Apesar disso, o consenso dos especialistas considerou que a qualidade dos registros do hospital estudado é compatível com o monitoramento de EAM por rastreadores, $\mathrm{e}$ que a técnica é útil para reconhecer problemas no processo de uso dos medicamentos.

$\mathrm{O} I H I^{9}(2004)$ indica a necessidade de 20 minutos, em média, para um prontuário ser revisado. Entretanto, neste estudo, a revisão ultrapassou esse tempo. Uma das explicações é o fato de que, embora os prontuários analisados apresentassem um ordenamento (exames laboratoriais, prescrições e evolução de enfermagem e médica), em muitos casos essas seções não se apresentavam na ordem cronológica dos acontecimentos, dificultando a análise.

A identificação da associação entre uso de medicamento e o EAM (causalidade) foi ponto crítico do processo, principalmente pela dificuldade em distinguir possíveis EAMs das complicações ou da evolução da própria doença. Entretanto, a reunião de consenso foi decisiva, pois nela os distintos profissionais - médico, farmacêutico e enfermeiro - apresentaram diferentes visões sobre cada situação, contribuindo para o estabelecimento da causalidade. Isso reforça a importância das comissões de farmacovigilância dentro do ambiente hospitalar, que podem ser articuladas para participar da implementação da técnica dos rastreadores.

A proposta do $I H I^{9}$ (2004) não inclui a aplicação de algoritmos ou de tabelas de decisão. O objetivo da avaliação de causalidade é estabelecer uma probabilidade para a suspeita de que um determinado fármaco seja responsável por um evento adverso. A avaliação de causalidade mais comumente usada em hospitais é o algoritmo de Naranjo $^{15}$, aqui aplicado e originalmente proposto para determinação de causalidade de reações adversas a medicamentos. Ele contribui para reduzir a subjetividade no julgamento e leva em conta, entre outros elementos, a literatura científica, a temporalidade, a história do paciente e as comorbidades.

A gravidade do paciente pode confundir a análise da associação entre o uso de medicamentos e a ocorrência de EAM. Na tentativa de emprego da gravidade como variável de ajuste, foram extraídas do prontuário informações sobre patologias pré-existentes ou diagnosticadas na internação. O Índice de Charlson ${ }^{21}$, método para classificação de gravidade usando dados de comorbidades, vem sendo aplicado para o ajuste de risco e foi demonstrada sua associação com a ocorrência de EAMs em pacientes ambulatoriais com idade igual ou superior a 65 anos $^{22}$. A determinação do Índice de Charlson não é condição indispensável para a aplicação da técnica; entretanto, é uma alternativa possível para o ajuste de risco.

\section{Considerações finais}

Os EAMs ocorridos no ambiente hospitalar são um problema reconhecido internacionalmente, daí a importância de identificá-los e monitorá-los com o objetivo de reduzir a sua ocorrência.

O objetivo primordial da ferramenta proposta pelo $I H I$ é monitorar a qualidade da atenção ao paciente, por meio da vigilância dos eventos adversos, do acompanhamento das estimativas ao longo do tempo, e do efeito da implantação das intervenções para reduzir os danos provocados pelos medicamentos em cada unidade assistencial.

Embora os rastreadores não sejam apropriados para comparar diferentes unidades, a técnica permite situar cada uma delas no conjunto da rede. Assim, seria recomendável a implantação da lista de rastreadores na rede hospitalar nacional, o que, aliado a outras técnicas de farmacovigilância, tais como a notificação espontânea, fomentaria o uso 
racional de medicamentos. No Brasil não há um amplo diagnóstico sobre o problema de EAM em hospitais; a aplicação da lista permitiria revelar diferenças e semelhanças entre os problemas encontrados nas diferentes unidades, o que complementaria os resultados de estudos epidemiológicos voltados ao cálculo de estimativas de prevalência e incidência, úteis para traçar perfis de morbidade associada ao uso de fármacos.

Finalmente, para o futuro, recomenda-se cuidado especial no processo de ajustes e adaptações dos rastreadores às condições locais. É importante também desenvolver, aprimorar e aplicar a ferramenta em outros contextos, tais como hospitais de especialidades, em unidades pediátricas e de terapia intensiva.

\section{Agradecimentos}

Ao Programa de Pós-Graduação em
Saúde Pública da Escola Nacional de Saúde Pública Sergio Arouca da Fundação Oswaldo Cruz, onde a primeira autora desenvolveu o estudo para obtenção do título de Doutora. Ao Dr. Cosme M.F.F. Passos da Silva, pelo apoio na seleção da amostra. Ao Dr. Evandro da Silva Coutinho, pela leitura crítica do texto na oficina de trabalho sobre a publicação de artigos científicos, organizada pelos programas de pós-graduação stricto sensu da ENSP e do IFF. À Dra Claudia M. R. Travassos e ao Dr Adriano Max Moreira Reis, pela leitura crítica e sugestões para o texto final. À farmacêutica Elaine Cristina Oliveira dos Santos e aos acadêmicos Caroline de Almeida, João Gabriel Nakka Strauch, Marlon Thiago Pereira e Michelen Critina Tonete, pela colaboração na etapa de coleta de dados. Ao CNPq, pelo apoio financeiro (Processo $n^{\circ}$ 471685/2007-0) e pela bolsa de doutorado concedida à primeira autora.

\section{Referências}

1. Baker GR, Norton PG, Flintoft V, Blais R, Brown A, Cox J et al. The Canadian Adverse Events Study: the incidence of adverse events among hospital patients in Canada. CMAJ. 2004; 170(11): 1678-86.

2. Mendes W, Martins M, Rozenfeld S, Travassos C. The assessment of adverse events in hospitals in Brazil. Int J Qual Health Care. 2009; 21(4): 279-84.

3. Cano FG, Rozenfeld S. Adverse drug events in hospitals: a systematic review. Cad Saude Publica 2009; 25(S3): 360-72.

4. Romero AV, Malone DC. Accuracy of adverse-drugevent reports collected using an automated dispensing system. Am J Health Syst Pharm 2005; 62(13): 1375-80.

5. Al-Tajir GK, Kelly WN. Epidemiology, comparative methods of detection, and preventability of adverse drug events. Ann Pharmacother 2005; 39(7-8): 1169-74.

6. Otero-López MJ, Alonso-Hernández P, MaderueloFernandez JA, Garrido-Corro B, Domínguez-Gil A, Sánchez-Rodríguez A. Acontecimientos adversos prevenibles causados por medicamentos en pacientes hospitalizados. Med Clin (Barc) 2006; 126(3): 81-7.

7. Otero MJ, Dominguez-Gil A. Acontecimientos adversos por medicamentos: una patologia emergente. Farm Hosp 2000; 24(4): 258-66.
8. Franklin BD, Birch S, Schachter M, Barber N. Testing a trigger tool as a method of detecting harm from medication errors in a UK hospital: a pilot study. Int J Pharm Pract. 2010; 18(5): 305-11.

9. Institute for Healthcare Improvement (IHI). Trigger tool for measuring adverse drug events. 2004. Disponível em http://www.ihi.org/NR/rdonlyres/8D970CE4BF8C-4F35-9BC1-51358FC8B43F/2222/

TriggerToolforMeasuringAdverseDrugEventsCorrected1. pdf. [Acessado em 7 de dezembro de 2010]

10. Roque HE, Melo ECP. Adaptação dos critérios de avaliação de eventos adversos a medicamentos para uso em um hospital público no Estado do Rio de Janeiro. Rev Bras Epidemiol 2010; 13(4): 607-19.

11 Rozenfeld S, Chaves SM, Reis LG, Martins M, Travassos $\mathrm{C}$, Mendes W et al. Adverse effects from drugs in a public hospital: pilot study. Rev Saude Publica 2009; 43(5): 88790 .

12. Resar RK, Rozich JD, Classen D. Methodology and rationale for the measurement of harm with trigger tools. Qual Saf Health Care 2003; 12(S2): 39-45.

13. Rozich JD, Haraden CR, Resar RK. Adverse drug event trigger tool: a practical methodology for measuring medication related harm. Qual Saf Health Care 2003; 12(3): 194-200. 
14. Committee of Experts on Management of Safety and Quality in Health Care (SP-SQS). Expert group on safe medication practices. Glossary of terms related to patient and medication safety. World Health Organization; 2005. Disponível em http://www.who.int/ patientsafety/highlights/COE_patient_and_medication_ safety_gl.pdf [Acessado em 7 de janeiro de 2011]

15. Naranjo CA, Busto U, Sellers EM, Sandor P, Ruiz I, Roberts EA, Janecek E, Domecq C, Greenblatt DJ. A method for estimating the probability of adverse drug reactions. Clin Pharmacol Ther 1981; 30(2): 239-45.

16. Lauritsen JM. (Ed.) EpiData Data Entry, Data Management and basic Statistical Analysis System. Odense Denmark, EpiData Association; 2000-2008. Disponível em http://www. epidata.dk [Acessado em 12 de junho de 2010].

17. Bellomo R, Ronco C, Kellum JA, Mehta RL, Palevsky P; Acute Dialysis Quality Initiative workgroup. Acute renal failure - definition, outcome measures, animal models, fluid therapy and information technology needs: the Second International Consensus Conference of the Acute Dialysis Quality Initiative (ADQI). Group Crit Care 2004; 8(4): R204-12.
18. Handler SM, Altman RL, Perera S, Hanlon JT, Studenski SA, Bost JE et al. A systematic review of the performance characteristics of clinical event monitor signals used to detect adverse drug events in the hospital setting. J Am Med Inform Assoc 2007; 14(4): 451-8.

19. Zegers M, de Bruijne MC, Wagner C, Groenewegen PP, van der Wal G, de Vet HC. The inter-rater agreement of retrospective assessments of adverse events does not improve with two reviewers per patient record. J Clin Epidemiol 2010; 63(1): 94-102.

20. Kilbridge PM, Campbell UC, Cozart HB, Mojarrad MG. Automated surveillance for adverse drug events at a community hospital and an academic medical center. $J$ Am Med Inform Assoc 2006; 13(4): 372-7.

21. Charlson ME, Pompei P, Ales KL, MacKenzie CR. A new method of classifying prognostic comorbidity in longitudinal studies: development and validation. $J$ Chronic Dis 1987; 40(5): 373-83.

22. Field TS, Gurwitz JH, Harrold LR, Rothschild J, DeBellis $\mathrm{KR}$, Seger AC et al. Risk factors for adverse drug events among older adults in the ambulatory setting. $\mathrm{J} \mathrm{Am}$ Geriatr Soc 2004; 52(8): 1349-54.

Recebido em: 05/05/11

Versão final apresentada em: 08/04/12

Aprovado em: 29/06/12 